\title{
NUTRIÇÃO DE EQÜINOS VIA ESOFAGOSTOMIA
}

\author{
NUTRITION OF HORSES VIA ESOPHAGOSTOMY
}

\author{
Marco Aurélio Ferreira Lopes ${ }^{1}$ Luiz Gonzaga Pompermayer ${ }^{2}$ \\ Aécio Henrique Barbosa Felipe ${ }^{3}$ Isabela Ciniello Araujo ${ }^{4}$
}

\section{- RELATO DE CASO -}

\section{RESUMO}

Cinco eqüinos com disfagia foram alimentados através de uma esofagostomia. Em dois eqüinos, o acesso ventral foi usado, enquanto nos outros o acesso foi ventrolateral. Em todos os casos, a extremidade da sonda foi posicionada no esôfago torácico. Uma dieta pastosa, composta de ração peletizada triturada, suplemento mineral e água, foi administrada através da sonda. Infecção periesofágica e complicações da doença primária foram as causas do óbito de dois eqüinos. Nos outros três casos, a evolução foi satisfatória. A sonda foi removida quando os eqüinos estavam aptos a se alimentar por via oral (depois de 15, 16 e 35 dias) e todos apresentavam condição corporal igual ou superior ao observado no dia do internamento. A abertura no esôfago cicatrizou por segunda intenção.

Palavras-chave: esofagostomia, eqüino, nutrição, disfagia, esôfago.

\section{SUMMARY}

Five horses presenting dysphagia were fed via an esophagostomy. In two cases, the ventral access was used while, in the others, the access was ventrolateral. In all cases, the tube tip was placed in the thoracic esophagus. A gruel composed of ground commercial pelleted feed, mineral supplement and water was given by the tube. Periesophagic infection and complications of the primary disease were the causes of the death of two horses. In the other cases the outcome was satisfactory. The tube was removed when ingestion could be resumed (after 15, 16 and 35 days) and all animals had a body condition equal or superior to that observed at admission. The esophageal opening healed by second intention.

Key-words: esophagostomy, horse, nutrition, dysphagia, esophagus.

\section{INTRODUÇÃO}

A esofagostomia pode ser usada como uma via para a nutrição enteral de eqüinos com disfagia. A técnica é relativamente simples e consiste na criação de uma abertura temporária no esôfago cervical e na introdução de uma sonda no segmento caudal do esôfago. Complicações, tais como extensa infecção periesofágica, hemiplegia laríngea e distúrbios digestivos, podem ocorrer, mas a nutrição via esofagostomia tem a grande vantagem de ter um baixo custo, pois permite a administração de uma dieta à base de alimentos normalmente utilizados para cavalos normais (FREEMAN \& NAYLOR, 1978, STICK et al., 1981). A administração dos alimentos por uma sonda naso-gástrica pode ser utilizada por alguns dias, mas vão ocorrer lesões nas fossas nasais e na faringe se esse procedimento for

${ }^{1}$ Professor Departamento de Veterinária, Universidade Federal de Viçosa (UFV), 36571-000, Viçosa, MG. E-mail: marlopes@mail.ufv.br. Autor para correspondência.

${ }^{2}$ Professor Departamento de Veterinária, UFV.

${ }^{3}$ Aluno de graduação em Medicina Veterinária, Departamento de Veterinária, UFV.

${ }^{4}$ Médico Veterinário, Rio de Janeiro, RJ. 
utilizado por um tempo prolongado. Outra alternativa seria o emprego de nutrição parenteral, que consiste na administração intravenosa de soluções estéreis contendo glicose, aminoácidos, lipídeos, vitaminas e minerais. Entretanto, essa técnica exige monitoração contínua e tem um elevado custo, que é proporcional ao peso do paciente e à duração do tratamento. A nutrição parenteral pode ter complicações como severos distúrbios metabólicos e tromboflebite (LEWIS, 1995). Vários estudos em medicina humana têm mostrado que a nutrição parenteral aumenta o risco de infecções e recomenda-se o uso da nutrição enteral sempre que possível (MAINOUS \& DEITCH, 1994). Considerando as particularidades da fisiologia digestiva dos eqüinos, manter o trato gastrointestinal inativo por um período prolongado, talvez, tenha um risco ainda maior nessa espécie. Este trabalho visa a relatar a utilização da esofagostomia como via para a nutrição enteral em cinco eqüinos com disfagia.

\section{RELATO DE CASOS}

Em cinco eqüinos, apresentando disfagia (Tabela 1), fez-se uma esofagostomia visando estabelecer uma via para a nutrição enteral. O diagnóstico da patologia primária e do estado geral dos pacientes foi feito com base na anamnese, no exame clínico e nos exames complementares (exame radiográfico, exame endoscópico e hemograma). Decidiu-se pela esofagostomia face à impossibilidade de se alimentar os equinos por via oral por pelo menos 10 dias. A esofagostomia foi feita no terço médio do pescoço em 4 casos, mas no cavalo com compactação do esôfago (caso 4) a cirurgia foi feita caudal à obstrução, na transição do terço médio para o terço caudal do pescoço. Nos 4 eqüinos que não apresentavam obstrução do esôfago (casos 1, 2, 3 e 5), uma sonda nasogástrica foi colocada antes da cirurgia para facilitar a identificação do esôfago. Independentemente da localização da esofagostomia, a técnica cirúrgica empregada foi semelhante em todos os casos. Os eqüinos foram sedados com romifidina $^{\mathrm{a}}$ e, após a tricotomia e a antissepsia da pele, fez-se a anestesia local com a injeção de lidocaína ${ }^{b}$ no tecido subcutâneo e na região periesofágica. Com um bisturi, fez-se uma incisão na pele com cerca de $7 \mathrm{~cm}$ na linha mediana ventral (quando o acesso era ventral) ou imediatamente ventral e paralela à jugular esquerda (quando o acesso era ventrolateral). Em seguida, fez-se a diérese romba do tecido subcutâneo e das fáscias, possibilitando a exposição do esôfago. Com um bisturi, fez-se uma incisão longitudinal, com cerca de $2 \mathrm{~cm}$, na adventícia e na parede muscular do esôfago. Usando-se uma tesoura, foi feita uma incisão longitudinal na submucosa e na mucosa

Tabela 1- História, sinais clínicos, diagnóstico, acesso cirúrgico para o esôfago e evolução pós-operatória de eqüinos apresentando disfagia alimentados via esofagostomia.

\begin{tabular}{|c|c|c|c|c|}
\hline Identificação & História e Sinais Clínicos & Diagnóstico & Acesso para o Esôfago & Evolução Pós-Operatória \\
\hline $\begin{array}{c}\text { Caso } 1 \\
\text { cavalo mestiço, } \\
10 \text { anos }\end{array}$ & $\begin{array}{l}\text { rigidez muscular notada há } 4 \\
\text { dias, tosse, presença de alimento } \\
\text { na traquéia e desidratação }\end{array}$ & tétano & $\begin{array}{l}\text { ventral no terço } \\
\text { médio do pescoço }\end{array}$ & $\begin{array}{l}\text { remissão da rigidez } \\
\text { muscular e retirada da } \\
\text { sonda após } 16 \text { dias }\end{array}$ \\
\hline $\begin{array}{c}\text { Caso } 2 \\
\text { égua mestiça, } \\
8 \text { anos }\end{array}$ & $\begin{array}{c}\text { disfagia e presença de } \\
\text { alimento nas narinas há } 10 \\
\text { dias, caquexia e desidratação }\end{array}$ & $\begin{array}{l}\text { paralisia } \\
\text { da faringe }\end{array}$ & $\begin{array}{c}\text { ventral no terço } \\
\text { médio do pescoço * }\end{array}$ & $\begin{array}{l}\text { infecção periesofágica, } \\
\text { mediastinite e óbito } \\
6 \text { dias depois }\end{array}$ \\
\hline $\begin{array}{l}\text { Caso } 3 \\
\text { potro Campolina, } \\
1 \text { ano }\end{array}$ & $\begin{array}{l}\text { rigidez muscular observada } \\
\text { há } 2 \text { dias e desidratação }\end{array}$ & tétano & $\begin{array}{l}\text { ventrolateral no terço } \\
\text { médio do pescoço }\end{array}$ & $\begin{array}{l}\text { decúbito e óbito } \\
\text { atribuídos ao tétano } \\
3 \text { dias depois }\end{array}$ \\
\hline $\begin{array}{l}\text { Caso } 4 \\
\text { cavalo mestiço, } \\
8 \text { anos }\end{array}$ & $\begin{array}{l}\text { disfagia e presença de } \\
\text { alimento nas narinas há } 15 \text { dias, } \\
\text { emagrecimento e desidratação }\end{array}$ & $\begin{array}{l}\text { compactação } \\
\text { do esôfago }\end{array}$ & $\begin{array}{l}\text { ventrolateral na transição } \\
\text { entre o terço médio e o } \\
\text { terço caudal do pescoço }\end{array}$ & $\begin{array}{l}\text { retirada da sonda e } \\
\text { deglutição normal } \\
\text { após } 15 \text { dias }\end{array}$ \\
\hline $\begin{array}{l}\text { Caso } 5 \\
\text { égua Mangalarga } \\
\text { Marchador, } 4 \text { anos }\end{array}$ & $\begin{array}{l}\text { delocamento lateral da } \\
\text { mandíbula notado após } \\
\text { trauma ocorrido há } 4 \mathrm{~h}\end{array}$ & $\begin{array}{l}\text { fratura } \\
\text { bilateral da } \\
\text { mandíbula }\end{array}$ & $\begin{array}{l}\text { ventrolateral no terço } \\
\text { médio do pescoço }\end{array}$ & $\begin{array}{l}\text { consolidação da fratura } \\
\text { e retirada da sonda } \\
35 \text { dias depois } * *\end{array}$ \\
\hline
\end{tabular}

* Foi feita uma dissecção mais extensa dos tecidos periesofágicos.

** Foi alimentada via sonda nasogástrica por 3 dias antes da esofagostomia. 
com o tamanho mínimo suficiente para a introdução da sonda. Em seguida, uma sonda ${ }^{c}$ com diâmetro externo de $17 \mathrm{~mm}$ e diâmetro interno de $11 \mathrm{~mm}$ foi introduzida na porção caudal do esôfago (Figura 1A). Medindo-se a distância entre a $13^{\mathrm{a}}$ costela e a esofagostomia, determinou-se a extensão da sonda a ser introduzida para posicionar sua extremidade no terço final do esôfago, próximo ao cárdia. Em seguida, a porção da sonda externa ao esôfago foi fixada ao cabresto com fita adesiva. No cavalo com compactação de esôfago (caso 4), foi necessário fazer a desobstrução da porção cranial do esôfago imediatamente antes de se introduzir a sonda. Para remover o alimento compactado, uma pinça de Foester foi introduzida pela esofagostomia no segmento cranial do esôfago. Nos eqüinos com a esofagostomia ventro-lateral (casos 3, 4 e 5), obser- vou-se que a porção externa da sonda se acomodava melhor ao pescoço, causando menos desconforto.

Os cuidados com a ferida cirúrgica restringiram-se à limpeza duas vezes ao dia com água limpa, colocando-se em seguida açúcar sobre a ferida. Nos eqüinos que não apresentavam tétano, fez-se a administração profilática de sulfadiazina e trimetoprina $^{\mathrm{d}}$ (30 mg/kg IM 12-12h) por 5 a 7 dias, iniciada $2 \mathrm{~h}$ antes da cirurgia. Os animais não imunizados contra tétano receberam também uma dose de vacina antitetânica ${ }^{\mathrm{e}}$ e soro antitetânico ${ }^{\mathrm{f}}$ (1500UI SC), além de uma segunda dose da vacina após 2 semanas. Os eqüinos com tétano já estavam sendo tratados com penicilina G procaína ${ }^{\mathrm{g}}$ (40000UI/kg IM 12-12h), vacina antitetânica ${ }^{e}$ e soro antitetânico ${ }^{\mathrm{f}}$ (20000UI IV e 20000UI intratecal) e nenhum outro medicamento foi administrado para a profilaxia de
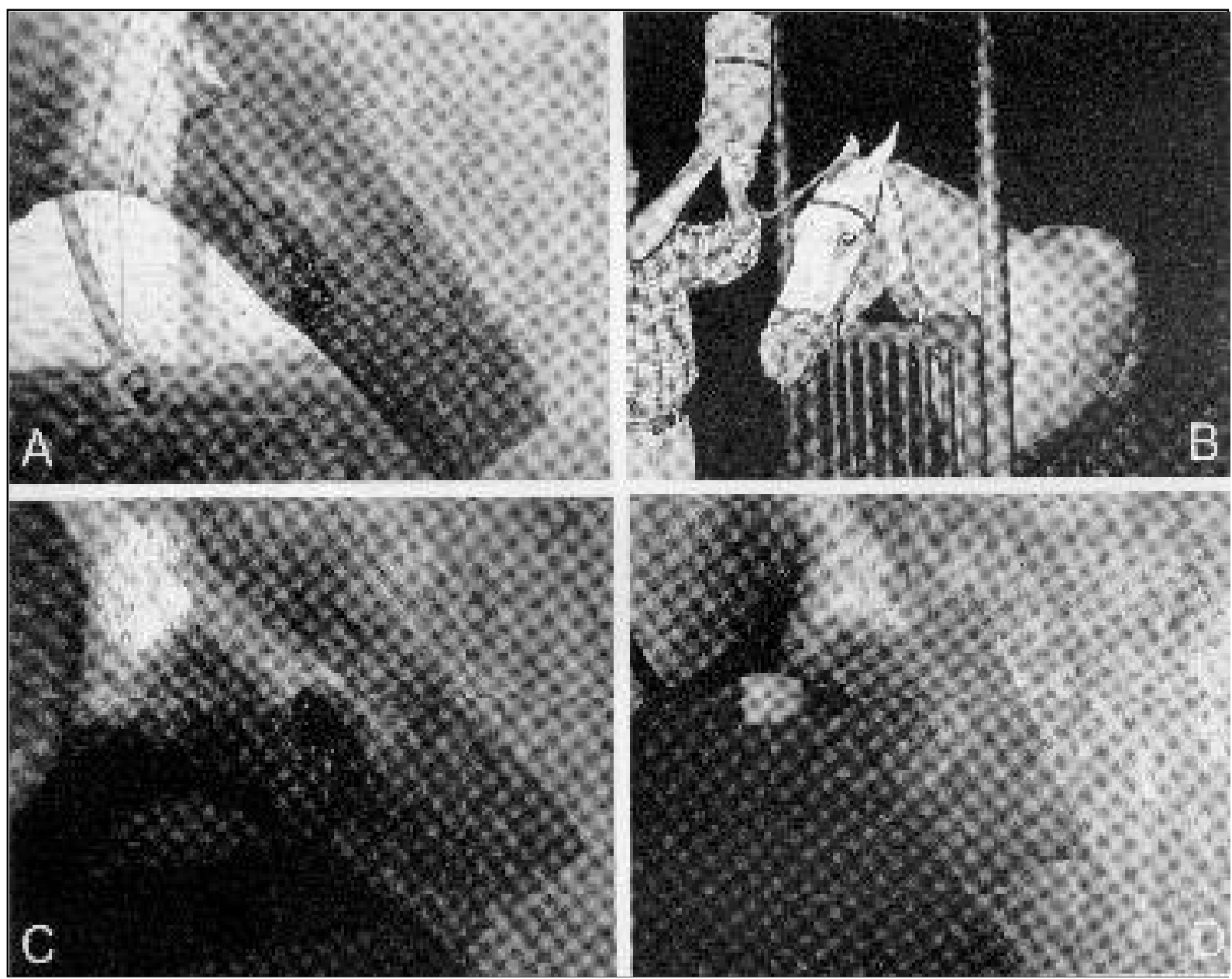

Figura 1 - Égua com fratura da mandíbula alimentada por 35 dias através de uma esofagostomia: A - Esofagostomia feita há 5 dias no terço médio do pescoço, com uma discreta reação inflamatória. B - Administração do alimento pastoso através da sonda. C - Aspecto da esofagostomia imediatamente após a retirada da sonda, mostrando o extravazamento de saliva. D - Face esquerda do pescoço, mostrando a completa cicatrização da esofagostomia, 3 semanas após a retirada da sonda.

Ciência Rural, v. 31, n. 1, 2001. 
infecções. Nos eqüinos que apresentavam sinais de desidratação (casos 1, 2, 3, 4), foram administrados nas primeiras $24 \mathrm{~h}$ aproximadamente $120 \mathrm{~m} \ell / \mathrm{kg}$ de água através da sonda.

A dieta foi calculada de acordo com o NRC (1989) para suprir $100 \%$ das exigências de energia e proteína para a mantença. $\mathrm{O}$ alimento era composto de ração comercial peletizada ${ }^{\mathrm{h}}$, triturada e suplemento mineral. Para a égua com fratura da mandíbula (caso 5), forneceram-se também feno de alfafa triturado $\left(2,4 \mathrm{~kg} /\right.$ dia) e óleo de soja ${ }^{\mathrm{j}}$. O alimento era dissolvido em 3 a 6 litros de água e, em seguida, era administrado através da sonda por gravidade (Figura 1-B). Freqüentemente, a sedimentação de partículas do alimento ocluiu a sonda. $\mathrm{Na}$ maioria dos casos, a desobstrução foi feita com a passagem de água sob pressão através da sonda, embora, algumas vezes, tenha sido necessário retirar a sonda para desobstruí-la. Após a administração do alimento, 0,5 $\ell$ de água eram administrados para remover todo o alimento da sonda e evitar o seu entupimento. Em todos os casos, a dieta foi gradativamente introduzida, de forma a alcançar $100 \%$ das exigências em 3 a 5 dias. A dieta era fornecida fracionada em 4 a 8 refeições e, entre as refeições, a sonda era mantida tampada.

Os eqüinos foram examinados a cada $12 \mathrm{~h}$. Visando a estimar o estado de hidratação, avaliaramse também o volume globular e a proteína plasmática total a cada $24 \mathrm{~h}$. O potro com tétano (caso 3 ) apresentou progressão da rigidez muscular, decúbito e óbito 3 dias após o início do tratamento. A égua com paralisia da faringe (caso 2) desenvolveu febre 2 dias após a cirurgia e morreu 4 dias depois. À necrópsia, não foi encontrada a causa da paralisia da faringe, mas observou-se extensa infeção periesofágica e mediastinite. Todos os eqüinos apresentaram inflamação na região da esofagostomia, mais evidente 24 a $48 \mathrm{~h}$ após a cirurgia. A inflamação, gradativamente, regrediu e no sétimo dia era mínima, quando se observou a formação de tecido de granulação e a contração da ferida. Prurido na região da esofagostomia, a partir de 10 dias da cirurgia, foi uma observação comum e, algumas vezes, os equiinos retiraram a sonda do esôfago friccionando-a na porta da baia durante a noite. A ausência do tubo resultou na rápida contração da esofagostomia, dificultando a reintrodução da sonda na manhã seguinte. Sobretudo nos primeiros dias, a deglutição resultava no extravazamento de saliva pela esofagostomia. Em todos os casos, notou-se uma regressão dos sinais de desidratação nas primeiras 24h. Enquanto eram alimentados pela esofagostomia, todos os eqüinos apresentaram fezes pastosas e poliúria, mas em nen- hum caso observou-se sinais de complicações decorrentes da dieta, como cólica ou laminite.

A sonda foi definitivamente retirada quando os eqüinos eram capazes de se alimentar por via oral. Naquele momento, todos apresentavam condição corporal e peso iguais ou superiores aos observados no dia do internamento. Nos primeiros dias após a retirada da sonda, notaram-se o extravazamento de saliva e alimento pela esofagostomia (Figura 1-C). Com a contração da ferida, o diâmetro da fístula e o volume de alimento que extravazava diminuíram rapidamente. Duas semanas após a retirada da sonda, a cicatrização estava completa em dois eqüinos (casos 1 e 4), mas, em um caso (5), o extravazamento de alimento ainda era observado e fez-se uma curetagem da fístula, que resultou no seu fechamento em 5 dias (Figura 1-D). A dieta originial à base de capim e grãos voltou a ser fornecida e não se observou nenhum sinal de mal funcionamento do esôfago por, pelo menos, 2 anos após a cirurgia. Um ano após a alta, um eqüino (caso 5) apresentou intolerância ao exercício, e hemiplegia laríngea esquerda foi diagnosticada por outro Médico Veterinário através de exame endoscópico. Nos outros animais, nenhum sinal sugestivo de patologia da laringe foi relatado e o exame endoscópico não foi realizado.

\section{DISCUSSÃO}

Tanto a técnica cirúrgica empregada, quanto a evolução pós-operatória observada nos 5 equiinos foram semelhantes ao descrito por FREEMAN \& NAYLOR (1978) e STICK $\boldsymbol{e t}$ al. (1981). Embora STICK et al. (1981) tenham recomendado o posicionamento da extremidade da sonda no estômago, optou-se por posicioná-la no segmento caudal do esôfago para evitar traumatizar o cárdia. Não se observou qualquer complicação com essa conduta, embora STICK et al. (1981) tenham relatado a ocorrência de refluxo de alimento ao redor da sonda quando a sua extremidade foi posicionada cranialmente ao cárdia. Quando a sonda foi precocemente removida para ser desobstruída ou pelo próprio animal, a reintrodução cuidadosa evitou complicações, embora STICK et al. (1981) tenham relatado a introdução acidental da sonda entre a mucosa e a parede muscular, quando a sonda precisou ser recolocada. O uso de uma sonda de grande calibre (17mm de diâmetro externo) não causou complicações e possibilitou a adição de feno de alfafa à dieta do animal tratado por 35 dias. Com a introdução gradual da dieta, não se observou cólica ou laminite. Entretanto CRAIG et al. (1989) relataram a ocorrência de laminite em animais com patologias do esôfago que voltaram a receber alimen- 
tação. A poliúria e a produção de fezes pastosas observadas em todos os casos, foram atribuídas ao grande volume de água necessário para fazer fluir o alimento pela sonda e ao baixo teor de fibras da dieta.

Não foi encontrado nenhum relato do uso da esofagostomia em eqüinos por um período tão longo como o ocorrido com a égua com fratura na mandíbula (35 dias). STICK et al. (1981) relataram a utilização experimental dessa técnica em cavalos normais por 30 dias. A demora na cicatrização da esofagostomia, observada no eqüino mantido com a sonda por 35 dias (caso 5), também foi relatada por STICK et al. (1981), em cavalos que permaneceram com a sonda por longo tempo. Eles atribuíram essa demora à excessiva fibrose do tecido de granulação. A infecção periesofágica e a mediastinite, observadas em um eqüino, também foram descritas por STICK et al. (1981), que atribuíram à contaminação dos tecidos periesofágicos antes da formação do tecido de granulação a causa dessa infecção. Na caso da égua com paralisia de faringe, considerou-se que a dissecção excessiva dos tecidos periesofágicos e o avançado estado de desnutrição tenham contribuído para a ocorrência da infecção.

A hemiplegia laríngea, diagnosticada após a alta no caso 5, pode ter sido causada pela cirurgia. Essa complicação foi relatada anteriormente em cavalos com patologias do esôfago (CRAIG $\boldsymbol{e t} \boldsymbol{a l}$., 1989) e em cavalos normais submetidos à esofagostomia (FREEMAN \& NAYLOR, 1978, STICK $\boldsymbol{e t}$ al., 1981). Entretanto, deve-se considerar que a hemiplegia laríngea pode ser idiopática (STICK et al., 1999). A possibilidade de um mal funcionamento da laringe antes da cirurgia também não pode ser excluída, pois não foi feito um exame endoscópico antes da cirurgia.

Concluiu-se que a esofagostomia permitiu a nutrição de eqüinos com disfagia por longos períodos e com baixo custo. Essa técnica possibilitou a nutrição por via enteral, que é a via fisiológica de nutrição, mantendo o trato gastrointestinal fun- cionante. Concluiu-se, também, que o acesso ventrolateral ao esôfago com mínima dissecção dos tecidos periesofágicos, o posicionamento da extremidade da sonda no esôfago torácico e a introdução gradual da dieta são indicados.

\section{FONTES DE AQUISIÇÃO}

a - Sedivet - Boerhinger De Angeli Química e Farmacêutica Ltda.

b - Lidocaína 2\% - Ariston Indústrias Químicas e Farmacêuticas Ltda.

c - Sonda Nasogástrica - Provar Comercial Ltda.

d - Tribrissen - Coopers Brasil S.A.

e - Vacina Antitetânica - Laboratório Hertape S.A.

f - Soro Antitetânico - Laboratório Hertape S.A.

$\mathrm{g}$ - Potencilin - Lema Biologic do Brasil Ltda.

h - Corcelina 200 - Purina Nutrimentos Ltda.

i - Suplemento Mineral Purina 80 - Purina Nutrimentos Ltda.

j - Óleo de Soja - Ceval Alimentos S.A.

\section{REFERÊNCIAS BIBLIOGRÁFICAS}

CRAIG, D., SHIVY, D.R., PANKOWSKI, R.L., et al Esophageal disorders in 61 horses results of nonsurgical and surgical management. Vet Surg, v.18, p.432-438, 1989.

FREEMAN, D.E., NAYLOR, J.M. Cervical esophagostomy to permit extraoral feeding of the horse. J Am Vet Med Assoc, v.172, p.314-320, 1978

LEWIS, L.D. Equine clinical nutrition. Baltimore : Williams \& Wilkins, 1995. Sick horse feeding and nutritional support: p.389-419.

MAINOUS, M.R., DEITCH, E.A. Nutrition and infection. Surg Clin North Am, v.74, n.3, p.659-676, 1994

NATIONAL RESEARCH COUNCIL. Nutrient requirement of Horses. 5.ed. Washington : National Academy of Sciences, 1989. 100p.

STICK, J.A., DERKSEN, F.J., SCOTT, E.A. Equine cervical esophagostomy: complications associated with duration and location of feeding tubes. Am J Vet Res, v.42, p.727-732, 1981.

STICK, J.A., TULLENERS, E.P., ROBERTSON, J.T., et al. Larynx. In: AUER, J.A., STICK, J.A. Equine surgery. 2.ed. Philadelphia : Saunders, 1999. p.349-368. 